\title{
Engineering Financial Performance Evaluation of Wireless Network Based on Intelligent Neural Network Model
}

\author{
Xiaoling $\mathrm{Xu}^{1}$ and Jianghao Song $\mathbb{D}^{2}$ \\ ${ }^{1}$ Guangdong Nanhua Vocational College of Industry and Commerce, Guangzhou, Guangdong, China \\ ${ }^{2}$ Guangdong Vocational Institute of Sport, Guangzhou, Guangdong, China \\ Correspondence should be addressed to Jianghao Song; songjh@nhic.edu.cn
}

Received 27 September 2021; Accepted 27 October 2021; Published 10 December 2021

Academic Editor: Narasimhan Venkateswaran

Copyright ( $) 2021$ Xiaoling Xu and Jianghao Song. This is an open access article distributed under the Creative Commons Attribution License, which permits unrestricted use, distribution, and reproduction in any medium, provided the original work is properly cited.

\begin{abstract}
Under the global economy, enterprises in the financial industry are facing plenty of opportunities and severe challenges. Aimed at providing a reference enterprise performance evaluation system for related enterprises, the proposed model helps enterprises to learn and sort out their own performance evaluation system according to this structure. A prediction model of BP neural network (BPNN) based on the wireless network is studied as the performance data prediction algorithm. Firstly, the feasibility of this algorithm is analysed through prediction training. Secondly, the proposed neural network algorithm is compared with the traditional algorithm for data prediction. It turns out that this neural network prediction algorithm based on wireless communication is not only universal to the prediction data but also superior to the traditional prediction algorithm in both error gap and relative average error compared with other traditional algorithms. On this basis, the particle swarm optimization (PSO) algorithm is also used to evaluate the performance indicators of three enterprises, and accurate numerical values are obtained to express the corresponding results. Therefore, it is concluded that the subalgorithm can be applied to the enterprise performance evaluation team in the financial industry.
\end{abstract}

\section{Introduction}

Since the 21st century, the global economy system has been gradually built. Because of the high-tech information technology and the rapid innovation of modern financial concepts, the financial markets all over the world have witnessed unprecedented changes, and various financial systems in it are not only facing variable opportunities but also bearing huge financial risks [1]. And with China's entry into the World Trade Organization, international competition has become more intense, so many financial institutions realize that it is the foundation of today's severe situation of the financial industry to focus on market dynamics [2]. Additionally, financial industries should adjust their management policies dynamically according to the market, to achieve real-time performance monitoring and improvement. Therefore, how to establish a set of enterprise performance evaluation institutions that can adapt to the environment and itself has become a research hotspot in recent years.
It is a very important job to evaluate financial institutions and the market situation objectively and practically, because the funds of financial institutions come from investors and customers. Merton and Thakor developed a framework to define the roles of customers and investors in the intermediary, used it to construct an economic theory, and studied the causes of risks and problems such as system design in the financial system [3]. Sezer and Ozbayoglu put forward a new algorithmic trading model, the Convolutional Neural Network-Trading Algorithmic, which used a twodimensional convolution neural network based on image processing characteristics and provided a better reference for stocks and Exchange-Traded Funds [4]. Additionally, the performance systems of other enterprises can provide some reference for themselves. Phan et al. comprehensively analysed the data of 228 companies listed in the Hanoi Stock Exchange and found that a good audit system played a positive role in securities trading, employee satisfaction, and customer loyalty [5]. Hosaka established a bankruptcy 
prediction model, using a convolutional neural network. Compared with the method using decision tree, linear discriminant analysis, support vector machine, multilayer perceptron, and AdaBoost's or Altman's Z-score, the trained network has higher performance in bankruptcy prediction [6]. Borovkova and Tsiamas proposed a set of long-shortterm memory (LSTM) neural networks for intraday stock forecasting using neural networks. And they used a variety of technical analysis indicators as network inputs. It was found that the proposed model performed better than the benchmark model or the same weighted ensemble [7]. $\mathrm{Li}$ et al. used piecewise graph convolution and recurrent neural network to classify the relationships in clinical notes without manual feature engineering. It indicates that adding syntactic dependency information helps to improve medical word embedding and conceptual relation classification without manually adding feature engineering [8]. Therefore, to establish a scientific and reasonable performance evaluation institution is undoubtedly to seize the dynamic trend of the market and the opportunity of success.

The performance evaluation of financial enterprises can be regarded as a process of finding the functional relationship between performance data. Because there are too many data models and influencing factors involved in the process and distinguishing the relationship between each variable is based on accurate evaluation, it is difficult to use ordinary mathematical equations to calculate. Therefore, the BP neural network (BPNN) based on the wireless network is adopted to solve the problem. BPNN can simulate the data of a complex nonlinear relationship. The debugged BPNN system is applied to the performance evaluation of enterprises, which not only makes the influence of human factors dissipate but also makes the evaluation more accurate. And BPNN is used to implement an evaluation model to predict enterprise performance, based on which, the PSO algorithm is added to evaluate the performance indicators of the three companies, to judge the availability of the model. The innovation of the experiment lies in the combination of a new PSO algorithm with the neural network, and the model is proposed for the enterprise performance evaluation.

\section{Materials and Methods}

2.1. Enterprise Performance Evaluation Theory. Performance evaluation has a long history. Since human beings took production activities, the idea of performance evaluation came into being. Hosaka studied the development history of performance evaluation with the method of literature [9], and the related research mentioned that "once human beings can carry out the production activities of goods needed for life and meet the needs of survival and reproduction, human beings begin to consider the comparison between cost and result." This comparison of cost and achievement is an early statement of performance evaluation. Therefore, it can be analysed that there may be the idea of performance evaluation in primitive society [10]. Performance evaluation mainly includes the following types:
(1) Asset value method [11]. It includes cost replacement, balance sheet, and market value. Cost replacement is the final value after the cost of an enterprise is replaced by mergers and acquisitions, and the related consumption and depreciation are removed. Market value is a validity hypothesis, which is based on the fluctuation of market price in macro conditions. The balance sheet is determined according to the amount of assets in traditional accounting books. It is a static valuation method, which is generally expressed in the balance sheet, as Figure 1.

(2) Relative valuation method [12]. As an indicator that can intuitively express enterprise value, the extended related indicators mainly express the ratio between enterprise value and sales profit. In the current huge market environment, the share is directly related to the profitability and operation effect of enterprises, and the market sales rate is an important standard for enterprises to go public and develop. This index is mainly used to evaluate the loss of enterprises, and its application scope is limited. The effect of capital utilization is the value of investors, as illustrated in Figure 2.

(3) Flow discount method [13]. It is a mainstream value method, which focuses on the cash and flow discount in the enterprise time period and depends on the future flow and advancement in the actual situation. In the 1980s, research experts defined and explained the flow of flowable funds. According to the error of payment objects of capital flow, capital flow can be divided into two types.

Enterprise capital flow value:

$$
V_{t}=\sum_{t=1}^{n} \frac{\mathrm{FCFF}_{t}}{(1+\mathrm{WACC})^{t}} .
$$

Share capital flow value:

$$
V_{t}=\sum_{t=1}^{n} \frac{\mathrm{FCFF}_{t}}{\left(1+K_{c}\right)^{t}} .
$$

In Equations (1) and (2), $V_{t}$ means the value, FCF stands for the free capital flow, WACC means the weighted average loss, $K_{C}$ refers to the cost, $F$ represents the fixed investment added when sales increase in 1 yuan, and $t$ accords to the time. These two types show different ways of handling funds.

Debt cost is [14] the cost incurred by the enterprise in financing, namely, the weighted average loss, which is calculated as shown in the following equation:

$$
\mathrm{WACC}=K_{b} \times \frac{B}{V} \times(1-T)+K_{s} \times \frac{S}{V} .
$$




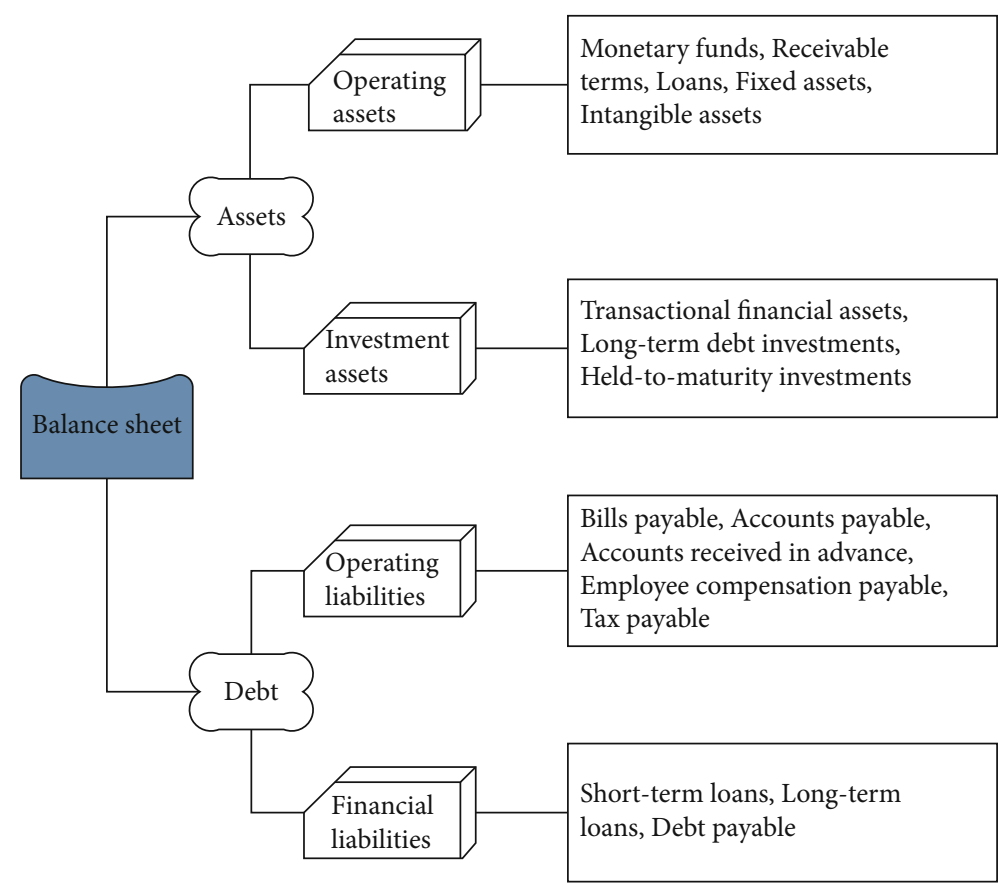

FIgURe 1: Asset value structure.
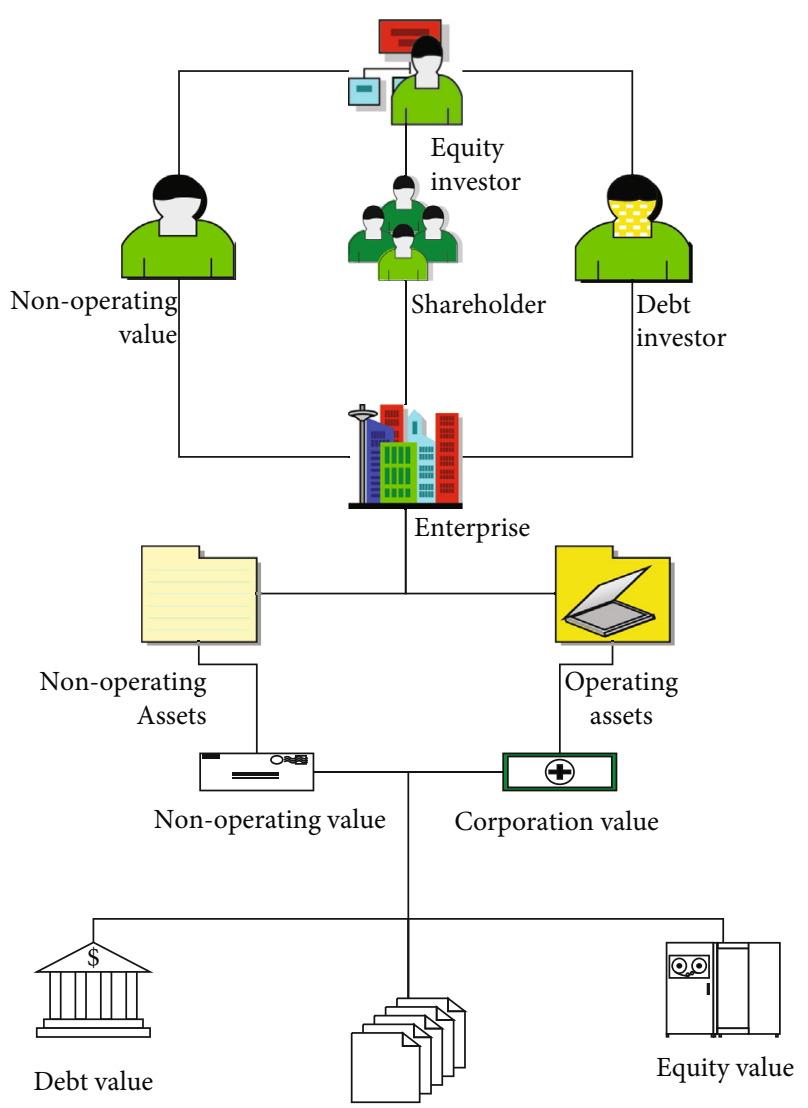

Value of minority shareholders' equity

FIgURE 2: Relative valuation structure.
In Equation (3), $K_{s}$ means the cost of shares, $K_{b}$ stands for the pretax cost, $S$ refers to the enterprise's share value, $B$ represents the enterprise's debt, and $V$ is the total assets of the enterprise.

From the standard of influencing capital flow, the enterprise applicable to the model must meet the following requirements: (1) discounting according to the capital flow in the future period; (2) income is the capital flow of the enterprise in recent years, and the risks can be controlled. Figure 3 shows the specific structure.

(4) Option valuation method [15]. The accuracy and universality have been improved through the research results of related concepts put forward by Western countries. In the 1970 s, a B-S model was put forward, the conceptual basis of which was the value of financial options. After optimization, the model was applied in practice, the actual enterprise valuation was explained, and the method and principle were expounded, whose structure is shown in Figure 4.

In the capital evaluation process, the widely used B-S model belongs to a binary tree model, and its calculation model is

$$
C_{0}=S_{0}\left[N\left(d_{1}\right)\right]-X e^{-r_{c} t}\left[N\left(d_{2}\right)\right]
$$

or expressed as

$$
C_{0}=S_{0}\left[N\left(d_{1}\right)\right]-P V(X)\left[N\left(d_{2}\right)\right]
$$




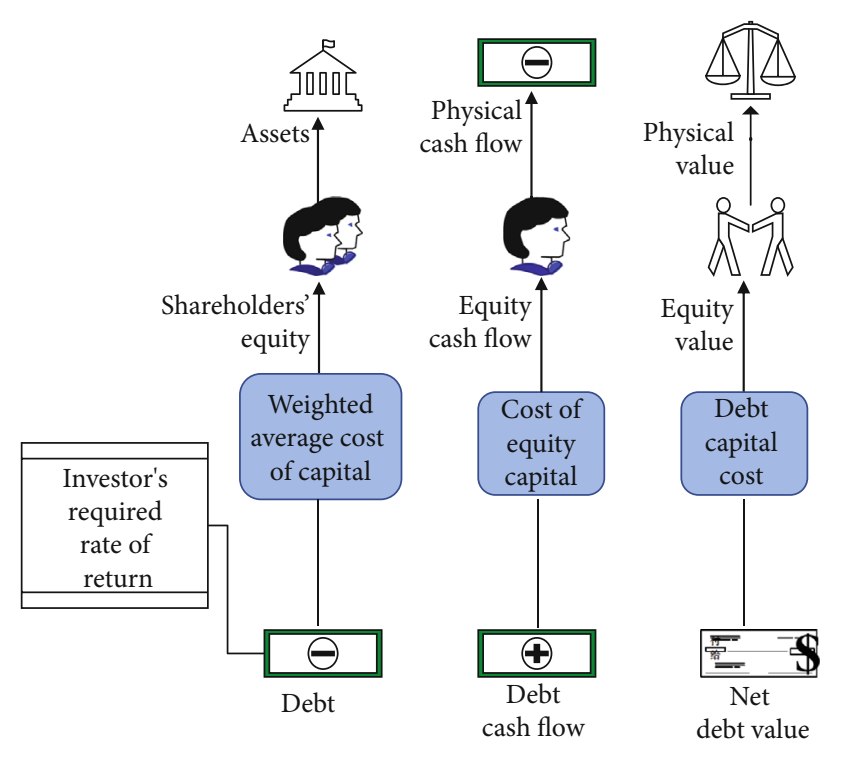

FIgURE 3: Flow discount structure.

$$
d_{1}=\frac{\ln \left(S_{0} / X\right)+\left[r_{c}+\left(\sigma^{2} / 2\right)\right] t}{\sigma \sqrt{t}}
$$

or expressed as

$$
\begin{gathered}
d_{1}=\frac{\ln \left[S_{0} / P V(X)\right]}{\sigma \sqrt{t}}+\frac{\sigma \sqrt{t}}{2}, \\
d_{2}=d_{1}-\sigma \sqrt{t} .
\end{gathered}
$$

In Equations (4)-(8), $C_{0}$ stands for the price when shares rise, $S_{0}$ means the current value of shares, $N\left(d_{1}\right)$ equals to the deviation less than $d$ in normal distribution, $X$ refers to the exercise value of options, $r_{c}$ stands for the continuous compound interest risk-free profit, $t$ represents the maturity time, and $\sigma^{2}$ means the variance of share return rate of continuous compound interest [16].

(5) Economic increase assessment method [17]. The traditional profit calculation tends to analyse the cost of enterprise debt, which usually has limitations, because financing of equity will also produce costs. However, Economic Value Added (EVA) can get rid of this limitation. When an enterprise is in a single-stage model, the enterprise can be guaranteed that it will develop relatively stable in a relatively mature stage. Every standard of the enterprise will not fluctuate greatly, so the EVA value of the enterprise at this stage is relatively stable. $g$ expresses the growth rate, and the calculation method is as follows:

$$
V=\frac{\mathrm{IC}+\mathrm{EVA}}{\mathrm{WACC}-\mathrm{g}}
$$

In Equation (9), EVA refers to the first EVA value in the period of steady growth.

When an enterprise passes the development stage, it will enter a new stage instead of steady growth, which can improve the accuracy of EVA evaluation again. Figure 5 shows the general EVA management system.

2.2. Neural Network Theory. The Artificial Neural Network (ANN) is a description of a certain stage of the human brain and a mathematical model, which is generally calculated by wireless network, and can also be calculated locally by computer. Its nature is a kind of artificial intelligence [18].

ANN is a complex nonlinear system, which consists of information-processing neuron units. Although the functions of individual neurons are simple, when a large number of neurons form a network, their functions are very complex. In recent years, various neural network models have been implemented in related research. Generally, there are three elements: neuron, neuron connection mode, and learning ability improvement mode [19]. Figure 6 explains the structure of a neural network in the human body.

The design of ANN also refers to the neural network of the human body, which is essentially a simplified model of the biological model. Related research has applied it in this field $[20,21]$, as Figure 7 demonstrates, which is the simulation of a single neuron unit.

The development process of the neural network is also from simple to complex. Figure 8 is the simplified structure of single nerve unit structure, which was put forward by Western researchers after they sorted out the algorithm of the two-layer neural network.

The two-layer neural network is the final goal of the study. At this stage, the neural network has a wide range of applications, but the single-layer neural network cannot simulate some problems. Due to the introduction of the computational layer, the two-layer neural network can make up for this shortcoming, and it can classify the data nonlinearly. Figure 9 displays the structure. In the 1980s, some scholars proposed a neural network including a propagation algorithm, which greatly improves the operation efficiency of neural network [22].

Multilayer neural network. On the basis of the two-layer structure, a new layer model is added again, as displayed in Figure 10. With the increase of layers, this structure can further show the data features and obtains a more accurate simulation function [23]. The first layer focuses on edge features, while the second layer focuses on edge shapes. In the third layer, the pattern features formed by shapes are simulated with emphasis, and different items are divided in this way, which can make the errors in features obvious.

2.3. Prediction Training of Neural Network. One of the advantages of a neural network is its ability to make an accurate calculation and certain prediction, which can learn and adapt to complex systems and data by itself. Therefore, compared with the statistical model, the neural network model has better accuracy [24]. Figure 11 illustrates the structural model of BPNN in wireless network communication. This model combines the advantages of a neural network and a 


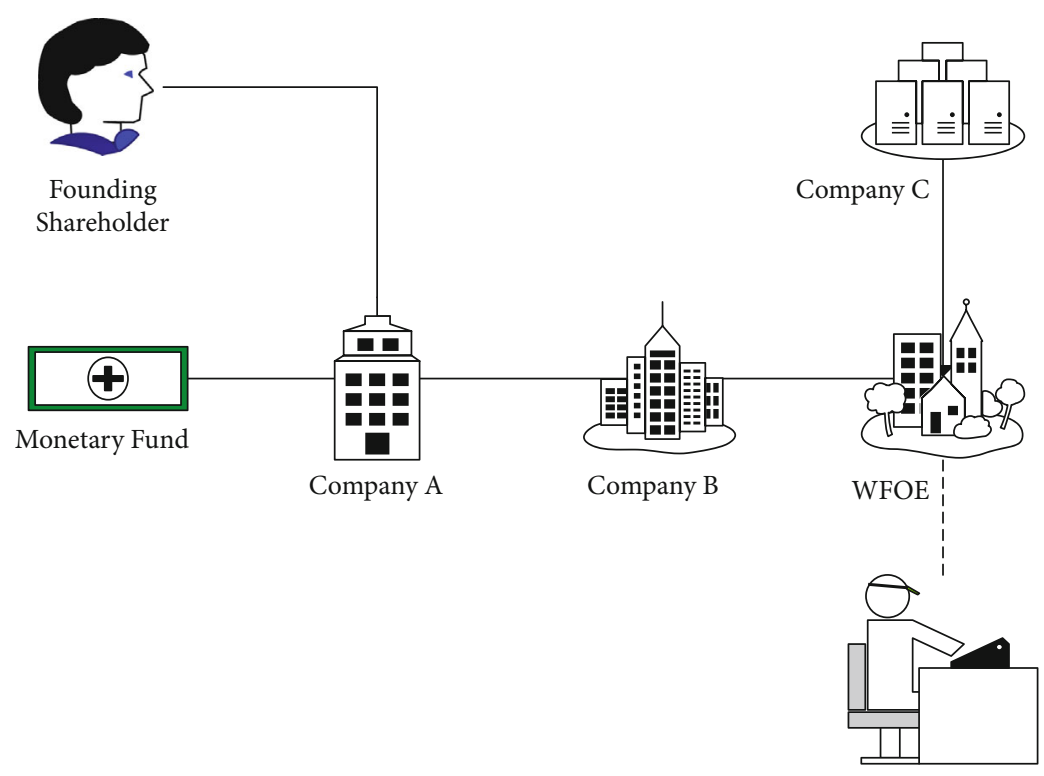

Founding shareholder monetary fund

Figure 4: Option valuation structure.

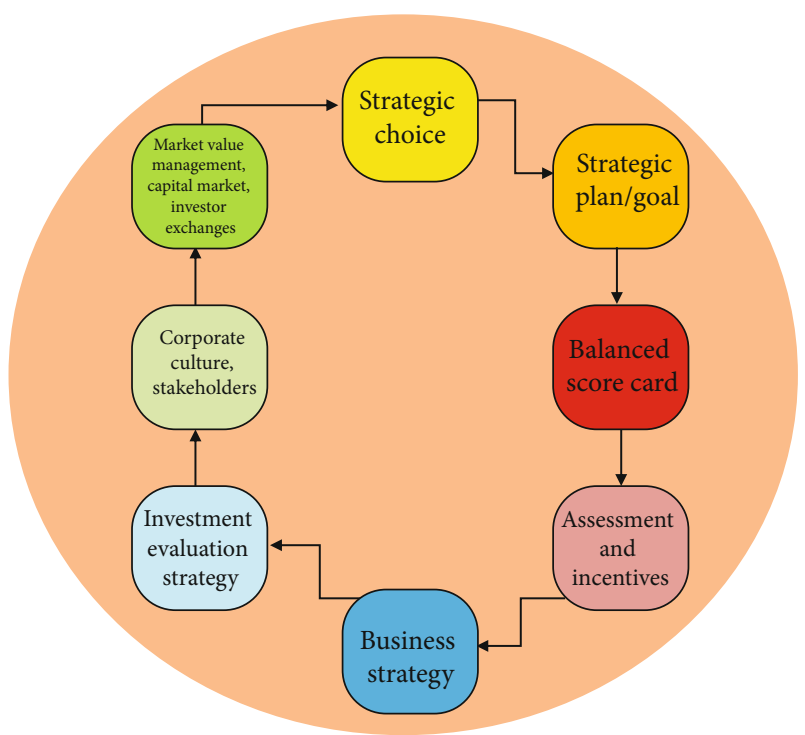

FIgUre 5: EVA evaluation system.

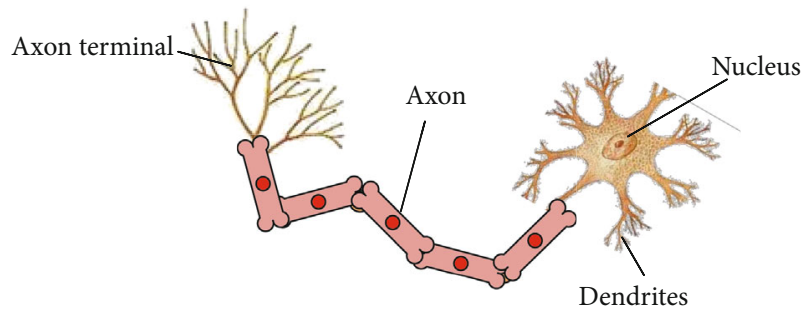

FIGURE 6: Human neuron structure.

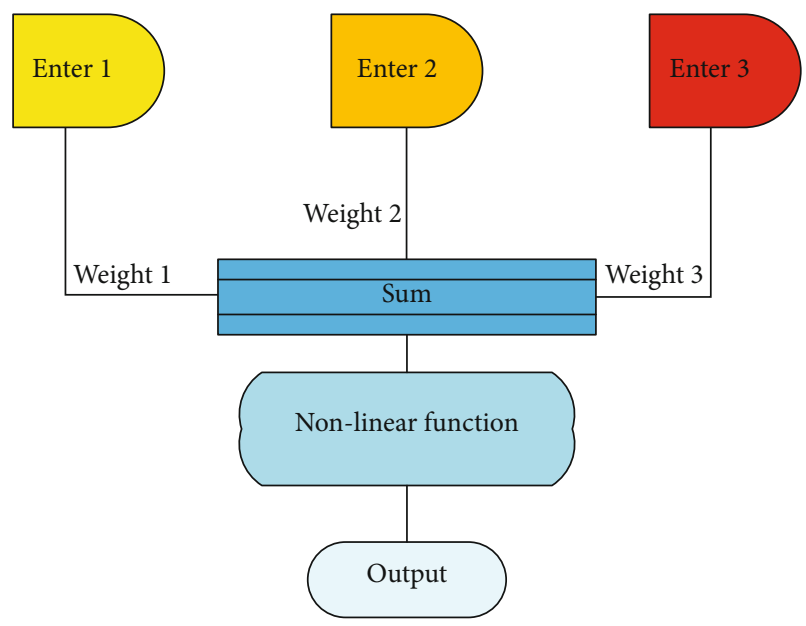

FIGURE 7: Single nerve unit structure.

wireless network and can better discover the variable relationship in a complex environment. In the beginning, the measured data can be divided into two types: training group and test group. The training group in the income layer can be either communication parameters or environmental variables, and the parameters in the output layer come from actual measurement or other models. Then, the training group is trained by the back-propagation principle in the hidden layer, and the fitting value is obtained in the output layer, which is further referred to as the communication model.

When training BPNN, the data must be unified [25], and the algorithm is used as follows:

$$
x^{\wedge}=\frac{2(x-\min (x))}{\max (x)-\min (x)}-1
$$




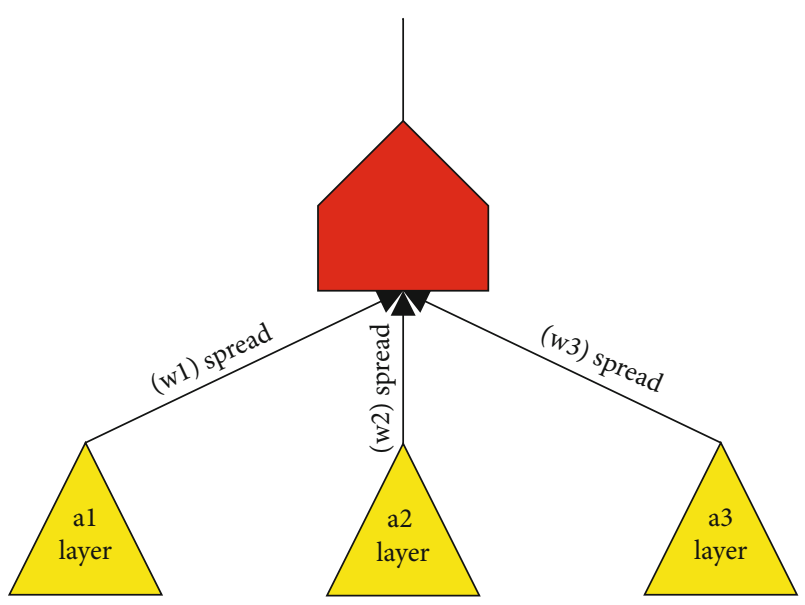

FIgURE 8: Simplified structure of single-layer neural network.

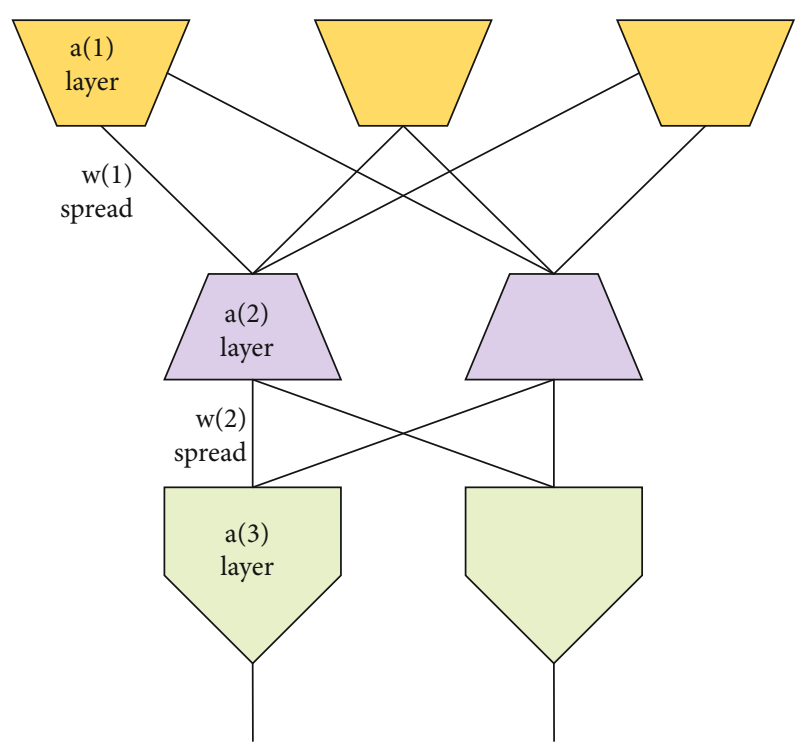

FIgURE 9: Two-layer neuron structure.

In Equation (10), the range of data can be located between [0,1]. After that, the unified data are processed in the order of income layer $\rightarrow$ hidden layer $\rightarrow$ output layer, and the output signal is sent out through nonlinear transformation. The neuron function of each layer uses the sigmoid function, which can be expressed as

$$
f(x)=\frac{1}{1+e^{-\left(W^{T} x+b\right)}} .
$$

Comparison is made between the actual value and the predicted value; if they are not the same, the total error e will be outputted in the network for reverse transmission. The output error is transferred in sequence: the output layer $\rightarrow$ hidden layer $\rightarrow$ income layer, and the residual error of nodes can be calculated in the following manner:

$$
W(t+1)=W(t)-\eta \frac{\delta E(t)}{\delta W(t)}
$$

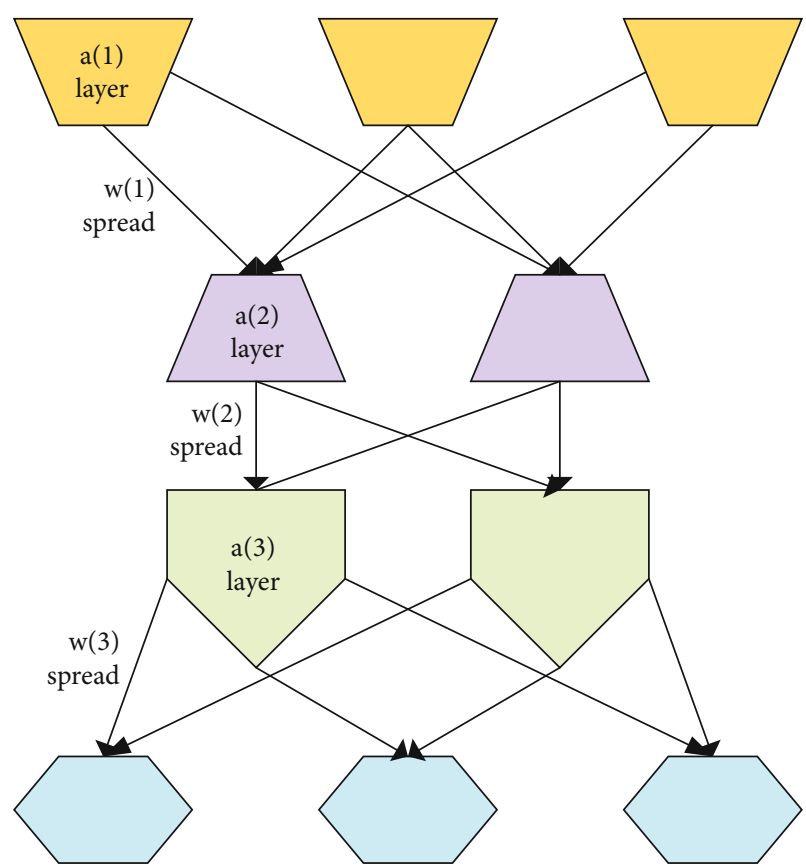

FiguRE 10: Three-layer neuron structure.

$$
b(t+1)=b(t)+\eta \frac{\delta E(t)}{\delta b(t)} .
$$

In Equations (12) and (13), $E(t)$ refers to the functional expression of total error, $t$ stands for the number of trainings, and $\eta \in(0,1)$ means the step factor.

$W=w^{(p) j i}$ means the network weight matrix, and $w^{(p) j i}$ stands for the connection between the $i_{\text {th }}$ nerve unit in the $p_{\text {th }}$ layer and the $j_{\text {th }}$ nerve unit in the $p_{\text {th }}$ layer. Parameter $x$ refers to the output vector, and the network threshold matrix $b=b^{(p) j}$ is the threshold of the $j_{\text {th }}$ nerve unit in the $p_{\text {th }}$ layer, displayed as

$$
\begin{gathered}
\frac{\delta E(t)}{\delta w_{j i}^{(p)}}=x_{j i}^{(p)} \sigma_{j}^{(p)}, \\
\frac{\delta E}{\delta b_{j}^{(p)}}=\sigma_{j}^{(p)}, \\
\sigma_{j}^{(p)}=f^{\prime} \cdot \sum_{k} w_{j k}^{(p+1)} \sigma_{k}^{(p+1)} .
\end{gathered}
$$

Among Equations (14)-(16), $\sigma_{j}^{(p)}$ stands for the $j_{\text {th }}$ neural unit error in layer $p$, and $f$ equals to the neural unit output, so the neural unit error in the output layer can be expressed as

$$
\sigma_{k}=f^{\prime}\left(x_{k}\right) \cdot\left[t_{k}-f\left(x_{k}\right)\right]
$$

By repeating the above training process, the error can be made smaller than the set threshold or close to the research 


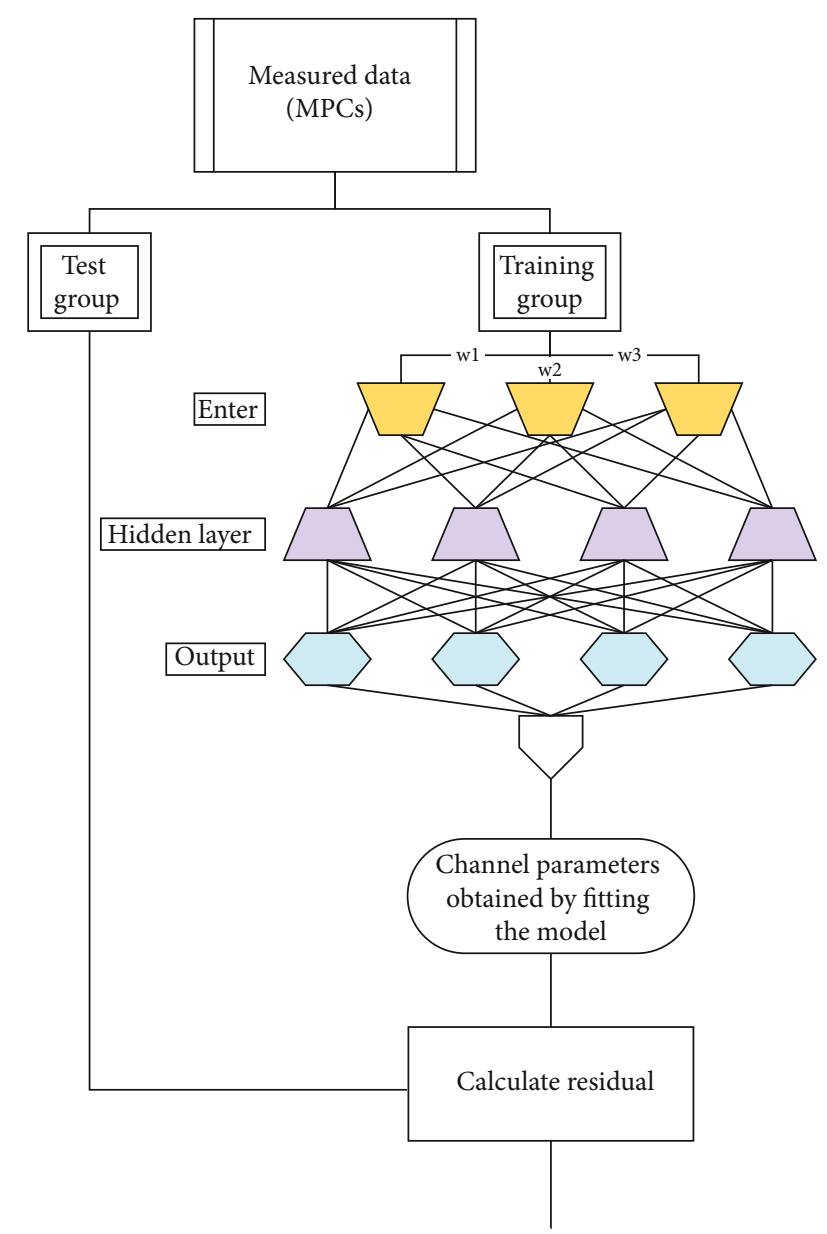

Figure 11: Structure of neural network in wireless communication.

requirement, and when it corresponds to the minimum error parameter, the training will be stopped.

2.4. BPNN in Enterprise Performance Prediction Model. When implementing the prediction model of BPNN, these aspects should be focused on: the number of neural networks, the number of neural units, and the rate of autonomous learning.

The more layers of the neural network, the more errors can be effectively reduced, and the higher the accuracy of calculation and prediction results can be obtained, because the BPNN model is adopted, which is usually with a three-layer structure. It is confirmed that the three-layer BPNN can achieve rational function, namely, multidimensional reflection [26]. On this basis, a hidden layer is added, and the number of neural units is increased to achieve improving the prediction accuracy.

In the process of designing the neural network prediction model, it is necessary to express certain correlations and set the relationship between the total market value and objective factors, which can be described by linear function as shown in the following equation:

$$
y=\sum_{j=1}^{M} x_{j} w_{j}+b .
$$

In Equation (18), $w_{j}$ means the weight and $b$ refers to the offset. These two coefficients represent the slope and intercept of the linear function image, respectively. Because the sigmoid function is used in the study, the function image is continuous and derivable. With reference to the characteristics of the function, the data with excessive gap can be limited to a certain interval, and the limited data change amplitude will not fluctuate again. Equation (19) displays the sigmoid function:

$$
f(z)=\frac{1}{1+e^{-z}}
$$

Primarily, samples of the loss function are required, and calculation is made on the error between the predicted value and the actual value of the neural network, to solve the coefficient matrix $w$ and the bias vector $b$. Then, the loss function is improved to obtain the minimum extreme value, and then, there are the bias vector $b$ and the matrix $w$. Because the loss function is used to calculate the gap value in the study, the predicted samples will be subject to gradient decline, which is expressed in the following equation:

$$
\text { MSE }=\frac{1}{N} \sum_{t=1}^{N}\left(\text { observed }_{t}-\text { predicted }_{t}\right)^{2} .
$$

MSE represents the mean square error [27, 28], which is the square expected value of the error between the predicted value and the true value calculated by parameters. Then, it is used to evaluate the data fluctuation level. Generally, the smaller the MSE, the more accurate the prediction of the data.

After being set, the prediction model has three layers in total. And each layer has a different number of neural units, including 6 income layers, 10 hidden layers, and 1 output layer. The sigmoid function is used, in which the value of the learning rate is set as 0.003 , and the loss function is expressed by MSE. Table 1 displays the relevant parameters of the model.

Several sections are repeated in the whole process. It is essential for the process to dynamically adjust the weights and functional thresholds of each layer through the direction of forward operation $\rightarrow$ loss calculation $\rightarrow$ backward operation.

2.5. Construction of Financial Performance Evaluation Model Based on BPNN. Primarily, the BPNN is initialized, and the initial values of each weight and threshold are set as random values between $(0,1)$ for later adjustment; then, the input value and expected output value of the sample training data are input, and the model is trained by the training sample data, which means that the actual output value and the output value of the hidden layer are obtained according to the input sample value, and the error between the expected output value and the actual output value is calculated. Then, there is the error between the output layer and the hidden layer. Afterwards, the connection weights and thresholds are adjusted according to the error, and finally, the error 
TABLE 1: Relevant parameters of neural network prediction model.

\begin{tabular}{lccc}
\hline $\begin{array}{l}\text { Model-related } \\
\text { parameters }\end{array}$ & $\begin{array}{c}\text { Input } \\
\text { layer }\end{array}$ & Hidden layer & $\begin{array}{c}\text { Output } \\
\text { layer }\end{array}$ \\
\hline Number of plies & 1 & 1 & 1 \\
Number of nerve units & 6 & 10 & 1 \\
Activate function & & Sigmoid & \\
Learning efficiency & 0.004 & 0.004 & 0.004 \\
Loss function & & MSE \\
End requirement & & MSE $=1 \times 10^{-4}$ \\
Batch size & & 15 \\
Optimization mode & & Adam \\
\hline
\end{tabular}

function is calculated. The global error is lower than the given error accuracy, indicating the network converge. Ultimately, the model training is completed. Figure 12 illustrates the workflow of the algorithm.

\subsection{Performance Evaluation Model of Air Traffic Control} Based on Factor Analysis and Particle Swarm Optimization (PSO) Algorithm. In the construction of the performance evaluation model of air traffic control, to evaluate the complex air traffic control system as objectively and comprehensively as possible, it will inevitably involve many indicators. Due to the fully connected Self-Organizing Feature Map (SOM) network structure, a large number of neurons in the input layer will lead to a complex network structure and slow convergence speed. With the advantages of factor analysis, the common ordered factor analysis is used as the input sample of the SOM neural network to improve the efficiency and reliability of the neural network. Meanwhile, the evaluation results are further refined to obtain the advantages and disadvantages of different elements within different evaluation objects, which is conducive to analysing the advantages and disadvantages of each evaluation object from different angles. The specific steps are as follows:

(1) There are two main purposes that can be reflected in the preliminary screening of experimental data. One is the adaptability test of data. The Kaiser-MeyerOlkin (KMO) and Bartlett Sphericity tests are used to ensure that the index system data have strong orthogonality. Factor analysis method can be used to prevent the loss of important data. When the $\mathrm{KMO}$ value is greater than 0.5 and the significance probability Sig value is less than 0.01 , it is proven that there are similar elements among the samples, and this method can be used for common factor extraction. The second is data normalization and standardization. Since the data come from different indicators, dimensions are different, and directions are different, it is necessary to standardize them into dimensionless data and positive data. The data standardization here is the standardization method, and the standardized data are expressed as $x_{i j}^{*}$ $(i=1,2, \cdots, n ; j=1,2, \cdots, m)$.
(2) The correlation matrix $R=\left(r_{i j}\right)_{\mathrm{mxm}}$ of the original indexes can be expressed as

$$
r_{i j}=\frac{1}{n-1} \sum_{k=1}^{n} x_{k i}^{*} x_{k j}^{*}=\frac{\sum_{k=1}^{n}\left(x_{k i}-x_{i}^{-}\right)\left(x_{k i}-x_{j}^{-}\right)}{\sqrt{\sum_{k=1}^{n}\left(x_{k i}-x_{i}^{-}\right)^{2}}\left(x_{k i}-x_{j}^{-}\right)^{2}} .
$$

(3) Parameter initialization of the PSO algorithm. According to the number of input nodes, namely, the number of common factors extracted by the factor analysis method for different ATC systems, the population size and related parameters are set. Initially, the number of populations is determined, which is usually $1-40.40$ particles are taken in the experiment. Then, the upper and lower bounds are set for speed $v$ and position $x$. The velocity will restrict the distance of the particle movement. When the velocity of the particle exceeds the upper or lower limit of the moving distance, the particle will reach the local optimum, so the optimal solution cannot be obtained. The value is usually the range width of the particle. Individual extremum $p_{i d}(0)$ and global extremum $p_{g d}(0)$ are initialized again. Consequently, the connection weights of the network are encoded, and the initial cluster of particles is obtained: $D$ means the number of connection weights (in Equation (23)) and $n$ and $q$ are the number of input layer nodes and the number of output layer nodes, respectively. Therefore, there are $D$ parameters that need to be optimized by the PSO algorithm; that is, the length of the particle is $D$ (in Eq. (23)). And, the particle $i$ is encoded with the following equation:

$$
\begin{aligned}
\operatorname{particle}(i) & =\left[w_{11}, w_{21}, \cdots, w_{n q}\right], \\
D & =n \times q .
\end{aligned}
$$

(4) The function which determines the fitness is the network distance function $d(y, w)$ of the particle swarm algorithm

$$
d(y, w)=\sqrt{\sum_{i=1}^{n}\left(y_{i}-w_{i j}\right)^{2}} .
$$

(5) The velocity and position of particles are updated according to Equations (25) and (26), and the updated particle fitness is calculated according to 


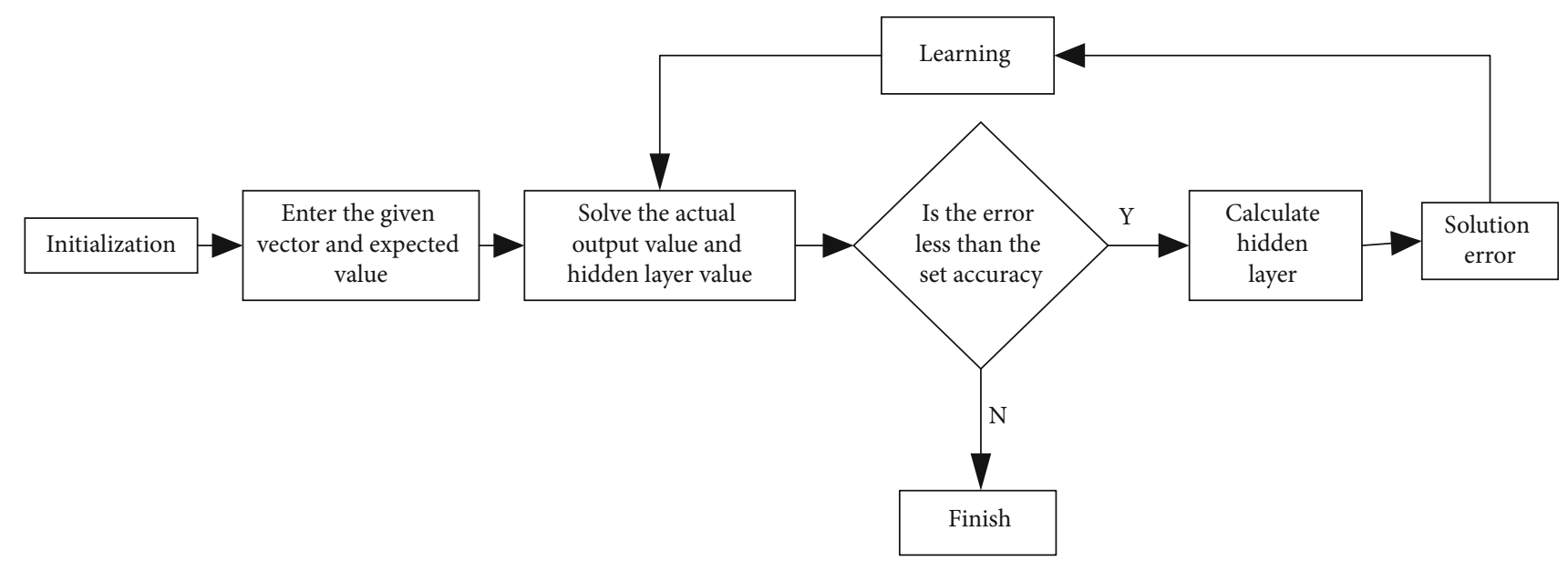

FIGURE 12: The workflow of the financial performance evaluation model based on BPNN.

Equation (24). The new particle fitness is compared with the individual extremum and global extremum of particles

$$
\begin{gathered}
v_{i d}(k+1)=w v_{i d}(k)+c_{1} r_{1}\left[p_{i d}(k)-x_{i d}(k)\right] \\
+c_{2} r_{2}\left[p_{g d}(k)-x_{i d}(k)\right] \\
x_{i}(k+1)=x_{i}(k)+v_{i}(k+1) \\
w(k)=w_{\max }-\frac{w_{\max }-w_{\min }}{k_{\max }} \times k .
\end{gathered}
$$

In Equations (25)-(27), $w$ refers to the inertia weight, $x_{i d}(k)$ represents the position of the current particle, $k$ means the number of iterations, $k_{\max }$ stands for the maximum number of iterations, $w_{\max }$ is values between 0.9 and $1.4, w_{\min }$ is the value 0.2 , and $c_{1}$ and $c_{2}$ refer to acceleration coefficients, which are generally nonnegative constants. There is a relationship between $c_{1}$ and $c_{2}: c_{1}=c_{2}=2$, and $r_{1}$ and $r_{2}$ are two random numbers, which $\in[0.1]$. Parameter $p_{i d}(k)$ refers to the individual extremum, and $p_{g d}(k)$ is the global extremum.

Figure 13 demonstrates the performance evaluation model based on the PSO algorithm.

\section{Results}

3.1. Error Curve Prediction. The prediction model of BPNN implemented is subjected to prediction training, input is made according to the set parameters, and the program is initialized for the prediction operation. When the prediction model is used for prediction training, after the program finishes the operation, the training prediction data are extracted and plotted into a graph, displayed as Figure 14.

The data in Figure 12 suggest that the prediction training of the model stopped after 2000 steps, but the result of prediction training did not reach the set value $4 \times 10^{-1}$, and the prediction training operation was terminated in advance, but the network prediction training process ended. Therefore, it means that the error is small between the result of prediction training using BPNN and the predicted value, and the model can be used to evaluate the performance of enterprises.

3.2. Comparison of Prediction Results between Traditional Valuation Method and Prediction Model of BPNN. In the experiment, seven companies were evaluated and forecasted for their performance, which are medical enterprise a, technology enterprise $b$, information enterprise $c$, network enterprise $d$, computer enterprise e, brokerage enterprise $f$, and Kexing enterprise g. Relative valuation method, cash flow forecasting method, B-S model forecasting, and EVA forecasting are used to forecast their performance value. The results are compared with the value predicted by BPNN, and analysation is made on the applicability and reliability of the model in enterprise performance evaluation.

The relative valuation method is used to forecast the above seven enterprises. Table 2 shows the results.

The analysis of the data in Table 2 signifies that the error between the performance value predicted by BPNN and the actual value is smaller than the value predicted by the relative valuation method, and the error of performance predicted by the neural network is about 498 million yuan at most and about 72 million yuan at least. The maximum error of the relative valuation method is about 1.882 billion yuan, and the minimum error is about 119 million yuan. Therefore, neural network prediction has better advantages compared with the relative estimation method.

On the same basis, the relative valuation method is changed to the cash flow forecasting method to evaluate the performance value, and the value of the above seven enterprises is predicted and compared with the neural network and test. Table 3 shows the data.

Analysis on the data in Table 3 reveals that the error between the cash flow valuation method and the actual value is close to the error between the predicted value and the neural network, so it is impossible to directly judge the accuracy. Therefore, the relative error method is adopted to judge. After calculation, the average value is -0.08556 of the relative error of the data predicted by the cash flow valuation method for the seven enterprises. The average relative error 


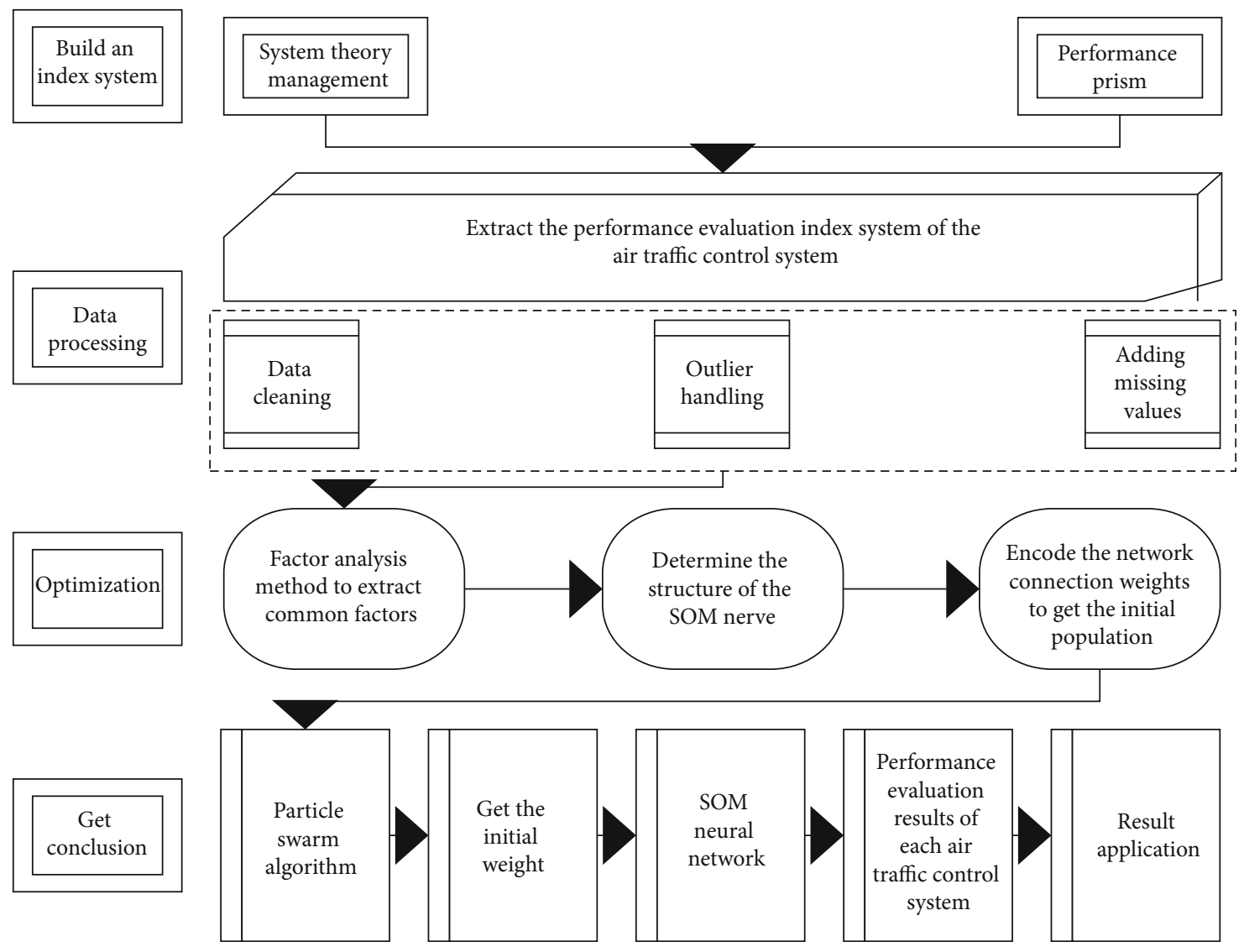

FIgURe 13: Performance evaluation model based on the PSO algorithm.

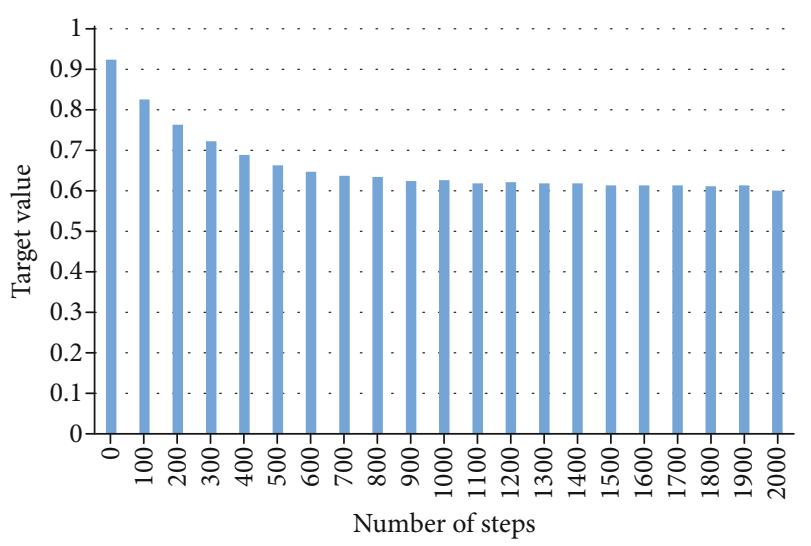

FigURE 14: Error prediction curve.

is 0.007193 of the neural network prediction data. By comparison, it is found that the neural network prediction method is obviously superior to the cash flow estimation method, which proves the accuracy and reliability of the neural network prediction method again.

Similarly, the B-S model is used to predict the performance value of the seven enterprises, and the data are collated and made into Table 4.

Table 4 suggests that the values of data predicted by the B-S model are all higher than those of the actual data, and the highest error value reaches 1.529 billion yuan, which may be due to subjective factors of parameter selection and other objective factors, resulting in the error value being higher than the actual value. Hence, the B-S model prediction method is not suitable for evaluating the performance value of enterprises. Once again, results prove the advantages of the neural network prediction method.

In the same way, the EVA prediction method is used to predict the performance value of the seven enterprises, whose results are shown in Table 5.

The comparison of the data indicates that the error between the EVA valuation data and the actual value is obviously larger than that of the neural network prediction method, and the maximum error has reached 2.711 billion yuan. The reason for the large error in EVA prediction is that it is difficult to find accurate data in books or reports, thus affecting the accuracy of the EVA prediction method. However, the accuracy of the prediction method of BPNN has been proven again.

3.3. Result Analysis of Simulation Based on BPNN. After the network model training is completed, that is, after the model is successfully constructed, what also needs to be verified is whether the constructed model is generalized. In other words, tests need to be made on whether it is suitable for the performance evaluation of ordinary data of newly listed energy companies. And simulation analyses also need to be carried out to observe the error accuracy between the simulation output results of its output layer and the expected 
TABLE 2: Error between relative valuation method and neural network prediction (data unit: 100 million yuan).

\begin{tabular}{lcccc}
\hline Enterprise code & Enterprise name & Data predicted by relative valuation method & Actual data & Data predicted by neural network \\
\hline 688109 & Medical enterprise a & 38.47 & 28.19 & 29.2089 \\
688169 & Technology enterprise b & 49.16 & 30.34 & 34.5409 \\
688388 & Information enterprise c & 27.82 & 26.63 & 25.9144 \\
688259 & Network enterprise d & 45.95 & 34.53 & 29.5557 \\
688039 & Computer enterprise e & 50.68 & 38.58 & 40.3049 \\
688389 & Brokerage enterprise f & 75.42 & 60.66 & 59.8516 \\
688009 & Kexing enterprise g & 127.57 & 136.25 & 134.89 \\
\hline
\end{tabular}

TABLE 3: Error between cash flow valuation method and neural network prediction (data unit: 100 million yuan).

\begin{tabular}{|c|c|c|c|c|}
\hline $\begin{array}{l}\text { Enterprise } \\
\text { code }\end{array}$ & Enterprise name & $\begin{array}{c}\text { Data of cash flow predicted by valuation } \\
\text { method }\end{array}$ & $\begin{array}{c}\text { Actual } \\
\text { data }\end{array}$ & $\begin{array}{c}\text { Data predicted by neural } \\
\text { network }\end{array}$ \\
\hline 688109 & Medical enterprise a & 26.36 & 28.19 & 29.2089 \\
\hline 688169 & $\begin{array}{c}\text { Technology enterprise } \\
\mathrm{b}\end{array}$ & 28.74 & 30.34 & 34.5409 \\
\hline 688388 & $\begin{array}{l}\text { Information enterprise } \\
\qquad \mathrm{c}\end{array}$ & 25.18 & 26.63 & 25.9144 \\
\hline 688259 & Network enterprise d & 31.77 & 34.53 & 29.5557 \\
\hline 688039 & Computer enterprise e & 35.55 & 38.58 & 40.3049 \\
\hline 688389 & Brokerage enterprise $f$ & 55.89 & 60.66 & 59.8516 \\
\hline 688009 & Kexing enterprise g & 127.57 & 136.25 & 134.89 \\
\hline
\end{tabular}

TABLE 4: B-S model prediction method neural network prediction gap (data unit: 100 million yuan).

\begin{tabular}{lcccc}
\hline Enterprise code & Enterprise name & Data predicted by B-S model & Actual data & Data predicted by neural network \\
\hline 688109 & Medical enterprise a & 31.49 & 28.19 & 29.2089 \\
688169 & Technology enterprise b & 39.69 & 30.34 & 34.5409 \\
688388 & Information enterprise c & 27.98 & 26.63 & 25.9144 \\
688259 & Network enterprise d & 38.77 & 34.53 & 29.5557 \\
688039 & Computer enterprise e & 43.21 & 38.58 & 40.3049 \\
688389 & Brokerage enterprise f & 62.36 & 60.66 & 59.8516 \\
688009 & Kexing enterprise g & 151.54 & 136.25 & 134.89 \\
\hline
\end{tabular}

TABle 5: Comparison between EVA prediction method and neural network prediction method (data unit: 100 million yuan).

\begin{tabular}{lcccc}
\hline Enterprise code & Enterprise name & Data predicted by EVA valuation method & Actual data & Data predicted by neural network \\
\hline 688109 & Medical enterprise a & 24.94 & 28.19 & 29.2089 \\
688169 & Technology enterprise b & 25.63 & 30.34 & 34.5409 \\
688388 & Information enterprise c & 27.62 & 26.63 & 25.9144 \\
688259 & Network enterprise d & 36.84 & 34.53 & 29.5557 \\
688039 & Computer enterprise e & 30.97 & 38.58 & 40.3049 \\
688389 & Brokerage enterprise f & 50.12 & 60.66 & 59.8516 \\
688009 & Kexing enterprise g & 109.14 & 136.25 & 134.89 \\
\hline
\end{tabular}

output results. From the sample data, 20 data reflecting the financial performance and 10 index data of evaluation are selected from the remaining data not participating in the training of the model as test data, namely, a $20 \times 10$ matrix as the input vector of BPNN, which are put into the BPNN model which has been trained to obtain the simulation results, as shown in Figure 15.

The two curves in Figure 15 demonstrate that the performance output value curve of the simulation sample basically coincides with the expected output value curve, and both 


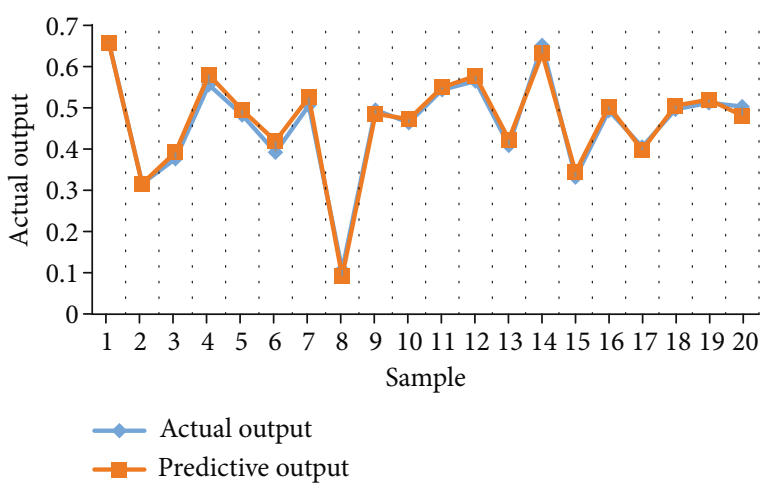

FIGURE 15: Comparison between the performance predicted by BPNN and the actual performance.

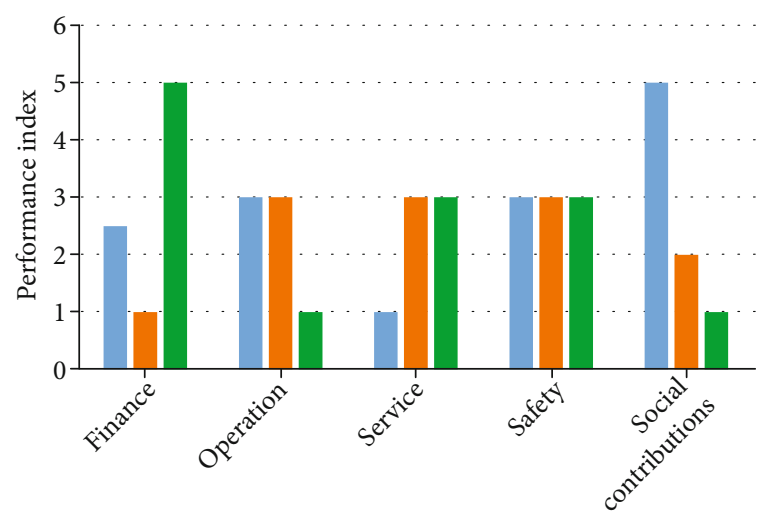

Performance

$$
\begin{aligned}
& \text { Company A } \\
& \text { Company B } \\
& \text { Company C }
\end{aligned}
$$

FIGURE 16: Results of the performance evaluation on 3 companies by performance evaluation model based on the PSO algorithm.

curves maintain the same trend. The actual output performance value of the $\mathrm{BP}$ prediction model is almost the same as the predicted performance value. Therefore, the performance evaluation model based on BPNN has better generalization and applicability and can be used to evaluate the financial performance of newly listed energy companies.

\subsection{Performance Evaluation Results of Performance} Evaluation Model Based on the PSO Algorithm. According to the performance evaluation model based on the particle swarm algorithm, evaluation is made on the performance of the three enterprises, and the financial, operational, service, security, and social contributions are evaluated, whose results are shown in Figure 16.

The performance evaluation model based on the particle swarm algorithm can clearly express the various performance indicators of the company through the number. The safety service of company A is at a very low level, but the index of contribution to society is very high. Company B's financial index is very low, but the development of other aspects is rather balanced; the financial index of company $\mathrm{C}$ is very high, while the contribution index to society is very low due to poor operation. Therefore, the operation of company $\mathrm{C}$ needs to be strengthened and reformed.

\section{Conclusions}

According to the background of the rapid development of global economy, discussion and studies are made on new performance evaluation systems of each enterprise in the financial industry. The study is aimed at providing some theoretical and algorithmic references for related enterprises and contributing to the financial enterprises adapting to the severe economic environment. Firstly, the concept of the enterprise's performance is expounded and studied based on the literature review. Secondly, the traditional performance evaluation methods are summarized. Thirdly, a BPNN algorithm is put forward based on wireless network information transmission. Fourthly, a prediction model is implemented based on the wireless neural network, and the prediction training is carried out. After the model is proven useful and versatile, it is used to evaluate the performance of seven enterprises. The results are compared with four traditional performance evaluation and prediction methods. Fifthly, it is found that the neural network prediction method is advantageous to the traditional prediction method in all aspects and has the advantages of high efficiency, high accuracy, and small fluctuation range. Therefore, the final conclusion is that the prediction model of BPNN, which can be applied to the enterprise performance evaluation in the financial industry, provides performance evaluation methods for all enterprises and companies and gives reference and optimization according to the neural network algorithm. Additionally, the BPNN and PSO algorithms are used to establish a performance evaluation model based on the neural network, whose performance values are predicted, and multiple performance indicators of three companies are evaluated. The performance evaluation model of BPNN can accurately predict the performance values of enterprises. The PSO evaluation model can intuitively express the performance of the enterprise through numerical values, indicating that both models can be applied to the performance evaluation of the enterprise. It sums up the performance evaluation theory applicable to enterprises, which can promote the whole financial industry. The improved and optimized performance evaluation model can evaluate the company's performance indicators in the business cooperation of enterprises, to reflect the operation ability and business ability of each company, and combine the model with other technologies, which can be applied in other industries, such as the evaluation of students' comprehensive performance or the physical index test of athletes. However, the limitation is on the use of the neural network algorithm to predict the performance value of enterprises and that an in-depth study is not made on the optimization of the enterprise performance evaluation. Therefore, in the follow-up research work, relevant literature will be learned, and more studies will be made on the optimization of performance evaluation. 


\section{Data Availability}

All data used are given in the paper.

\section{Conflicts of Interest}

The authors declare that they have no conflicts of interest.

\section{References}

[1] P. Sethi, D. Chakrabarti, and S. Bhattacharjee, "Globalization, financial development and economic growth: perils on the environmental sustainability of an emerging economy," Journal of Policy Modeling, vol. 42, no. 3, pp. 520-535, 2020.

[2] C. Baden-Fuller and D. J. Teece, "Market sensing, dynamic capability, and competitive dynamics," Industrial Marketing Management, vol. 89, no. 89, pp. 105-106, 2020.

[3] R. C. Merton and R. T. Thakor, "Customers and investors: a framework for understanding the evolution of financial institutions," Journal of Financial Intermediation, vol. 39, no. 39, pp. 4-18, 2019.

[4] O. B. Sezer and A. M. Ozbayoglu, "Algorithmic financial trading with deep convolutional neural networks: time series to image conversion approach," Applied Soft Computing, vol. 70, no. 70, pp. 525-538, 2018.

[5] T. Phan, L. Lai, T. T. le, D. M. Tran, and D. T. Tran, "The impact of audit quality on performance of enterprises listed on Hanoi Stock Exchange," Management Science Letters, vol. 10, no. 1, pp. 217-224, 2020.

[6] T. Hosaka, "Bankruptcy prediction using imaged financial ratios and convolutional neural networks," Expert Systems with Applications, vol. 117, pp. 287-299, 2019.

[7] S. Borovkova and I. Tsiamas, "An ensemble of LSTM neural networks for high-frequency stock market classification," Journal of Forecasting, vol. 38, no. 6, pp. 600-619, 2019.

[8] Y. Li, R. Jin, and Y. Luo, "Classifying relations in clinical narratives using segment graph convolutional and recurrent neural networks (Seg-GCRNs)," Journal of the American Medical Informatics Association, vol. 26, no. 3, pp. 262-268, 2019.

[9] L. dos Santos Matos, S. M. I. Valmorbida, V. A. Martins, and S. R. Ensslin, "Development of performance evaluation theme: a systematic analysis of the literature," Contextus-Revista Contemporânea de Economia e Gestão, vol. 17, no. 2, pp. 63-97, 2019.

[10] X. Islami, E. Mulolli, and N. Mustafa, "Using management by objectives as a performance appraisal tool for employee satisfaction," Future Business Journal, vol. 4, no. 1, pp. 94-108, 2018.

[11] C. Ghosh, M. Liang, and M. T. Petrova, "The effect of fair value method adoption: evidence from real estate firms in the EU," The Journal of Real Estate Finance and Economics, vol. 60, no. 1-2, pp. 205-237, 2020.

[12] H. Dukalang, "Analysis of stock intrinsic value using relative valuation on agriculture sector listed in JII70 period 20162019," Mutanaqishah: Journal of Islamic Banking, vol. 1, no. 1, pp. 50-58, 2021.

[13] A. Jumran and R. Hendrawan Sr., "Stock valuation using discounted cash flow method with free cash flow to equity and relative valuation approaches on state-owned banks listed on IDX for 2021 to 2025 period projection," International Journal of Science and Management Studies (IJSMS), no. 4, pp. 191201, 2021.
[14] M. Cowling, E. Ughetto, and N. Lee, "The innovation debt penalty: cost of debt, loan default, and the effects of a public loan guarantee on high-tech firms," Technological Forecasting and Social Change, vol. 127, no. 127, pp. 166-176, 2018.

[15] J. Zhao, "American option valuation methods," International Journal of Economics and Finance, vol. 10, no. 5, p. 1, 2018.

[16] M. N. Anwar and L. S. Andallah, "A study on numerical solution of Black-Scholes model," Journal of Mathematical Finance, vol. 8, no. 2, pp. 372-381, 2018.

[17] N. Tikasari and D. A. Surjandari, "The effect of economic value added and financial performance on stock return," Saudi Journal of Business and Management Studies SSN, pp. 2415-6663, 2020.

[18] Y. Wu and J. Feng, "Development and application of artificial neural network," Wireless Personal Communications, vol. 102, no. 2, pp. 1645-1656, 2018.

[19] A. Taherkhani, A. Belatreche, Y. Li, G. Cosma, L. P. Maguire, and T. M. McGinnity, "A review of learning in biologically plausible spiking neural networks," Neural Networks, vol. 122, no. 122, pp. 253-272, 2020.

[20] U. Hasson, S. A. Nastase, and A. Goldstein, "Direct fit to nature: an evolutionary perspective on biological and artificial neural networks," Neuron, vol. 105, no. 3, pp. 416-434, 2020.

[21] M. Mohammadi, Y. H. Tan, W. Hofman, and S. H. Mousavi, "A novel one-layer recurrent neural network for the $l_{1}$-regularized least square problem," Neurocomputing, vol. 315, no. 315, pp. 135-144, 2018.

[22] A. Javanmard, M. Mondelli, and A. Montanari, "Analysis of a two-layer neural network via displacement convexity," The Annals of Statistics, vol. 48, no. 6, pp. 3619-3642, 2020.

[23] H. Zhou, G. Sun, S. Fu, J. Liu, X. Zhou, and J. Zhou, “A big data mining approach of PSO-based BP neural network for financial risk management with IoT," IEEE Access, vol. 7, no. 7, pp. 154035-154043, 2019.

[24] G. M. Lozito and A. Salvini, "Swarm intelligence based approach for efficient training of regressive neural networks," Neural Computing and Applications, vol. 32, no. 14, pp. 10693-10704, 2020.

[25] F. Zhu, R. Gong, F. Yu et al., “Towards unified INT8 training for convolutional neural network," in 2020 IEEE/CVF Conference on Computer Vision and Pattern Recognition (CVPR), pp. 1969-1979, Seattle, WA, USA, 2020.

[26] H. S. Jo, C. Park, E. Lee, H. K. Choi, and J. Park, "Path loss prediction based on machine learning techniques: principal component analysis, artificial neural network, and Gaussian process," Sensors, vol. 20, no. 7, p. 1927, 2020.

[27] M. A. Jafari and A. Aminataei, "Some applications of sigmoid functions," Iranian Journal of Numerical Analysis and Optimization, vol. 11, no. 1, pp. 221-233, 2021.

[28] Y. Ye, Z. Wang, and X. Zhang, “An optimal pointwise weighted ensemble of surrogates based on minimization of local mean square error," Structural and Multidisciplinary Optimization, vol. 62, no. 2, pp. 529-542, 2020. 